\title{
Effects of the food colorant carmoisine on zebrafish embryos at a wide range of concentrations
}

\author{
Tuba Kiziltan $^{1,2} \cdot$ Alper Baran $^{3} \cdot$ Meryem Kankaynar $^{1,2} \cdot$ Onur Şenol $^{4} \cdot$ Ekrem Sulukan $^{1,5} \cdot$ Serkan Yildirim $^{6}$. \\ Saltuk Buğrahan Ceyhun ${ }^{1,5}$
}

Received: 28 December 2021 / Accepted: 27 January 2022 / Published online: 10 February 2022

(c) The Author(s), under exclusive licence to Springer-Verlag GmbH Germany, part of Springer Nature 2022

\begin{abstract}
Since the middle of the twentieth century, the use of dyes has become more common in every food group as well as in the pharmaceutical, textile and cosmetic industries. Azo dyes, including carmoisine, are the most important of the dye classes with the widest color range. In this study, the effects of carmoisine exposure on the embryonic development of zebrafish at a wide dose scale, including recommended and overexposure doses (from 4 to $2000 \mathrm{ppm}$ ), were investigated in detail. For this purpose, many morphological and physiological parameters were examined in zebrafish exposed to carmoisine at determined doses for $96 \mathrm{~h}$, and the mechanisms of action of the changes in these parameters were tried to be clarified with the metabolite levels determined. The no observed effect concentration (NOEC) and median lethal concentration $\left(\mathrm{LC}_{50}\right)$ were recorded at $5 \mathrm{ppm}$ and $1230.53 \mathrm{ppm}$ dose at $96 \mathrm{hpf}$, respectively. As a result, it was determined that the applied carmoisine caused serious malformations, reduction in height and eye diameter, increase in the number of free oxygen radicals, in apoptotic cells and in lipid accumulation, decrease in locomotor activity depending on the dose and at the highest dose, decrease in blood flow rate. In the metabolome analysis performed to elucidate the metabolism underlying all these changes, 45 annotated metabolites were detected.
\end{abstract}

Keywords Carmoisine $\cdot$ E122 $\cdot$ Food additive $\cdot$ Metabolome $\cdot$ Dyes

\section{Introduction}

Dyes are included in the class of important pollutants due to their main components called chromophores and auxochromes (Micheletti et al. 2020; Sohrabi et al. 2017). Its annual global production is estimated to be around 1 million

Saltuk Buğrahan Ceyhun saltukceyhun@hotmail.com

1 Aquatic Biotechnology Laboratory, Faculty of Fisheries, Atatürk University, Erzurum, Turkey

2 Department of Nanoscience, Graduate School of Natural and Applied Science, Atatürk University, Erzurum, Turkey

3 Department of Food Quality Control and Analysis, Technical Vocational School, Atatürk University, Erzurum, Turkey

4 Department of Analytical Chemistry, Faculty of Pharmacy, Atatürk University, Erzurum, Turkey

5 Department of Aquaculture, Faculty of Fisheries, Atatürk University, 25240 Erzurum, Turkey

6 Pathology, Faculty of Veterinary, Atatürk University, Erzurum, Turkey tonnes and more than 2.000 structurally different dyes are currently used (Elbanna et al. 2017). Azo dyes containing at least one $-\mathrm{N}=\mathrm{N}-$ group have a wide range of colors and are the most important synthetic materials of the dye classes (Kiayi et al. 2019). Carmoisine (disodium-4-hydroxy-3-[(4sulfo-1-naphthalenyl)azo]-1-naphthalenesulfonate) in this group is an anionic food dye with a color scale from red to burgundy (Ai-Mashhedy and Fijer 2016). Carmoisine is also known by different names such as Azorubine, Food Red 3, Azorubin S, Brillantcarmoisin O, Acid Red 14, and CI 14720. The permits and regulations for its use in food differ from country to country. For example, it is banned in the USA and Japan, it is free to use with ADI (acceptable daily intake) in European Union (Peksa et al. 2015). Highly water-soluble carmoisine is an organic azo dye that is widely used in food products such as jams, marzipan, jellies, yogurts, breadcrumbs, and cheesecake mixes, as well as in the pharmaceutical, textile and cosmetic industries (Amin et al. 2010). Due to this highly water-soluble feature, it does not degrade by exposure to light and oxygen, and as a result, it can be a permanent pollutant, especially in the aquatic 
environment (Micheletti et al. 2020). Due to this potential pollutant feature, many studies have been reported in which various methods have been tried to remove carmoisine from aquatic environments (Kiayi et al. 2019).

The azo dye carmoisine can be reduced to aromatic amines via the intestine by the microbiota and azoreductase in the liver or intestinal wall. Sulfanilic acid is the main metabolite formed as a result of these reactions (Chung et al. 1992). Nitrite compounds, which can be found in foods, combine with certain amino acids and then turn into carcinogenic N-nitroso compounds or nitrosamines. In this respect, International Agency for Research on Cancer has classified azo dyes such as carmoisine as category 3 carcinogens (Reza et al. 2019). In addition, EFSA and finally FDA shared their concerns about the use of food dyes, especially for children, who are common consumer groups. In addition, it has been reported that carmoisine cause to liver damage and reduced some metabolic enzymes in rats (Amin et al. 2010), genotoxicity by causing chromosomal aberrations and decreased mitotic index in Allium cepa (Khan et al. 2020), carcinogenicity and biochemical toxicity by reducing the expression of some important defense genes in mice (Reza et al. 2019). On the other hand, according to the best knowledge we have, the effects of carmoisine on metabolism have not been investigated in a metabolomics perspective, which is one of the most modern approaches to observe important changes that may be caused by endogenous metabolites. Therefore, in this study, the toxic effects of carmoisine on a wide dose scale were investigated by metabolic methods on a modern model organism, zebrafish. Zebrafish is a unique model organism with its rapid developmental stages, which are widely used in both adult and embryo stages in toxicological studies, allowing easy monitoring of embryogenesis and evaluation of any developmental defects due to the transparency of the egg and embryo, and its $70 \%$ similarity to the human genome (Baran et al. 2018; Capriello et al. 2021; Sökmen et al. 2020). Thus, in our current study, the effects of carmoisine on zebrafish embryos were evaluated in terms of morphological, molecular, behavioral, circulatory system, and metabolomics.

\section{Materials and methods}

\section{Embryo treatment and morphologic and physiologic measurements}

The ancestors of $\mathrm{AB}$ strain zebrafish (Danio rerio) larvae exposed to carmoisine in the study were obtained from Oregon State University. Embryos from rootstocks that had developed normally and reached the blastula stage were exposed graded concentrations of carmoisine $(4,50$, $100,200,400,800,1200,1600$, and $2000 \mathrm{ppm}$ ) which was prepared in E3 embryo medium. The control group was kept in E3 medium alone. All mediums were renewed every $24 \mathrm{~h}$. The experiment for each analysis was conducted with three independent experiments $(n=3)$ and each compound-treated group contained minimum 40 embryos. During the $96-\mathrm{h}$ exposure, the fish were examined under the microscope, and dead fish and hatching rates were recorded every $24 \mathrm{~h}$. In addition, morphological abnormalities (included pericardial edema, yolk sac edema, curved body axis, and head malformations) observed every $24 \mathrm{~h}$ during the exposure period were recorded. Morphological observation, survival and hatching rates were determined as a result of examining at least 200 embryos larvae (with 3 biological replicates, 600 fish in total) from each group. The median lethal concentration $\left(\mathrm{LC}_{50}\right)$ and no observed effect concentration (NOEC) were calculated according to Organization for Economic Co-operation and Development (OECD) guideline for testing chemical toxicity (OECD 2013). For eye size and body length measurements, 20 larvae (with 3 biological replicates, 60 larvae in total) were randomly selected from each group pool. For heart rate and blood flow, 10 larvae (with 3 biological replicates, 30 fish in total) randomly selected from each group pool were anaesthetized with tricaine. Videos of $3 \mathrm{~min}$ were recorded in a suitable format for software from the pericardiac and blood vessel regions of anesthetized larvae under the stereomicroscope. Video records were transferred to DanioScope and the software automatically calculated the number of beats per second/min (BPS/BPM) in the heart and measured the flow activity in blood vessels determined.

\section{Whole mouth larval staining}

Detection of apoptotic cells was performed using acridine orange, a nucleic acid selective metachromatic dye that interacts with DNA and RNA from 5 randomly selected 96 hpf (hours post-fertilization) larvae (3 replicates, in total 15 larvae) from each group from larvae exposed to carmoisine. Analysis was performed according to the standard protocol; in brief, the $96 \mathrm{hpf}$ larvae were first washed three times with PBS, then incubated with acridine orange at a concentration of $5 \mathrm{mg} / \mathrm{mL}$ at $28.5{ }^{\circ} \mathrm{C}$ for $30 \mathrm{~min}$. in the dark. After incubation, they were washed three times with PBS and examined under fluorescence microscopy (Zeiss, Discovery V12, Germany). Detection of reactive oxygen species (ROS) was performed using CM-H 2 DCFDA, a molecule that reacts with many different ROS and it is a general indicator of oxidative stress, from 5 randomly selected 96 hpf larvae (3 replicates, in total 15 larvae) from each group from larvae exposed to carmoisine. Analysis was performed according to the standard protocol; in brief, the larvae were first washed three times with ultra-pure $\mathrm{H}_{2} \mathrm{O}$, then incubated with CM- $\mathrm{H}_{2}$ DCFDA at a concentration of $1 \mu \mathrm{g} / \mathrm{mL}$ at 28.5 ${ }^{\circ} \mathrm{C}$ for $2 \mathrm{~h}$ in the dark. After incubation, they were washed 
three times with ultra-pure $\mathrm{H}_{2} \mathrm{O}$ and examined under fluorescence microscopy (Zeiss, Discovery V12, Germany). Finally, detection of lipid droplets in larvae body was performed using Nile Red, a compound that can interact with intracellular lipid droplets, from 5 randomly selected 96 hpf larvae (3 replicates, in total 15 larvae) from each group from larvae exposed to carmoisine. Analysis was performed according to the standard protocol; in brief, the larvae were first washed three times with ultra-pure $\mathrm{H}_{2} \mathrm{O}$, then incubated overnight with Nile Red at a concentration of $10 \mathrm{ng} / \mathrm{mL}$ at $28.5^{\circ} \mathrm{C}$ in the dark. After incubation, they were washed three times with ultra-pure $\mathrm{H}_{2} \mathrm{O}$ and examined under fluorescence microscopy (Zeiss, Discovery V12, Germany). Fluorescence intensity analysis of the obtained images was done with the ImageJ software (Baran et al. 2020; Türkoğlu et al. 2021).

\section{Larvae locomotor activity analysis}

The locomotor activity of zebrafish larvae exposed to carmoisine was evaluated by standard protocol. For this purpose, to monitor the activities of 24 zebrafish larvae (with 3 biological 72 larvae in total) randomly selected from each group pool with an infrared analog camera (25 frames per second), the 24-well plate containing one larva (6 dpf) in each well was placed in the DanioVision Observation Chamber (Noldus Co.). The temperature condition of observation chamber was carried out at $28{ }^{\circ} \mathrm{C}$ using a heating/cooling system (Noldus Co.). Dark/light periods were applied to create locomotor activity in response to changing dark and light conditions. Following 10-min habitation in dark, 10-min light and 10-min dark conditions were applied for images to be recorded for a total of $50 \mathrm{~min}$ (Sulukan et al. 2021). Monitoring and evaluation of the behavior of zebrafish larvae was carried out using the EthoVision software (Noldus Co.). The analyses were repeated by three independent replications.

\section{Metabolomic analysis}

To evaluate the effects of Carmoisine on the embryonic development period of zebrafish at the metabolite level, metabolite levels were determined from 25 larval pools from each group of zebrafish exposed to $50 \mathrm{ppm}$ and $2000 \mathrm{ppm}$ concentrations. Six pools were created for each concentration and the larvae were homogenized in $1 \mathrm{~mL}$ of methanol with the aid of TissueLyser (Qiagen). After that, homogenized samples were exposed to ultrasonic wave for $10 \mathrm{~min}$ and then incubated at $-20{ }^{\circ} \mathrm{C}$ for 15 min to initiate cell lysis and elevate the extraction efficacy. The mixtures have centrifuged at 10,000 rpm for $10 \mathrm{~min}$ and the supernatants were picked up and then evaporated under the vacuum (Eppendorf Concentrator+). The residues were reconstituted by $80 \mu \mathrm{L}$ of mobile phase mixture $(0.1 \%$ formic acid-water, $50: 50$, v/v). Quality control samples (QCs) were pooled by collecting
$5 \mu \mathrm{L}$ of aliquots from each sample. QCs were applied after every five consecutive sets to normalize batch drift and remove false peaks.

The total run time for Q-TOF MS/MS analysis was about 15 min for each sample and QC. Linear gradient elution was carried out in which the flow rate is $400 \mu \mathrm{l} / \mathrm{min}$. Conditions summarized as follows: $95 \%$ of mobile phase $\mathrm{A}\left(\mathrm{H}_{2} \mathrm{O}(0.1 \%\right.$ $\mathrm{v} / \mathrm{v}$ Formic acid)) were arranged to be $0.5 \mathrm{~min}$, followed by an elevation from 2 to $20 \%$ for mobile phase $\mathrm{B}\left(\mathrm{CH}_{3} \mathrm{CN}\right.$ $(0.1 \% \mathrm{v} / \mathrm{v}$ Formic acid) $)$ in $3.5 \mathrm{~min}$ and then $20 \%$ to $95 \% \mathrm{~B}$ in $4 \mathrm{~min}$. Conditions of $95 \%$ solvent B were held for $1 \mathrm{~min}$ and a 0.25 min gradient was used to return to the initial conditions, which were held for $2.75 \mathrm{~min}$. The total run time was about 15 min for each run.

\section{Histopathological analysis}

The zebrafish larvae exposure to carmoisine were detected in $4 \%$ paraformaldehyde solution for $48 \mathrm{~h}$. Five larvae from each group (15 larvae in 3 replications in total) were used. The samples were embedded in paraffin blocks after routine tissue tracking. $4 \mu \mathrm{m}$ thickness sections were taken from each block. Preparations prepared for histopathological examination were stained with hematoxylin-eosin (HE) and examined with a light microscope (Olympus BX 51, Japan). Sections were evaluated according to their histopathological features as none, very mild, mild, moderate, severe, and very severe.

\section{Results and discussion}

To assess the developmental toxicity effects of carmoisine on zebrafish embryo/larvae, survival and hatching rates were determined. The NOEC was calculated at $5 \mathrm{ppm}$ on the carmoisine-induced mortality curve of zebrafish embryos (Fig. 1B). The $\mathrm{LC}_{50}$ estimated at $96 \mathrm{hpf}$ was $1230.53 \mathrm{ppm}$ (Supplementary file). As shown in Fig. 1, high concentration exposure of carmoisine significantly increased the mortality ( $<$ 0.05) (Fig. 1B). Similarly, it was observed that exposure to carmoisine caused a decrease in early hatching rates dose-dependent manner (Fig. 1C). A possible explanation for the delayed hatching in our experiment may be that the embryos observed in some eggs could not break out the chorion. Another reason may be the inhibition of enzymes involved in hatching triggered by the dye (Baran et al. 2018). Another explanation for difficulty hatching is neuromuscular deficiencies that cause weakening of spontaneous muscle movements (De la Paz et al. 2017). As a matter of fact, a decrease in hatching rate was observed in zebrafish embryos exposed to different toxicants including azo dyes (Gupta et al. 2019; Joshi and Katti 2018; Li et al. 2017; Suvarchala and Philip 2016). 

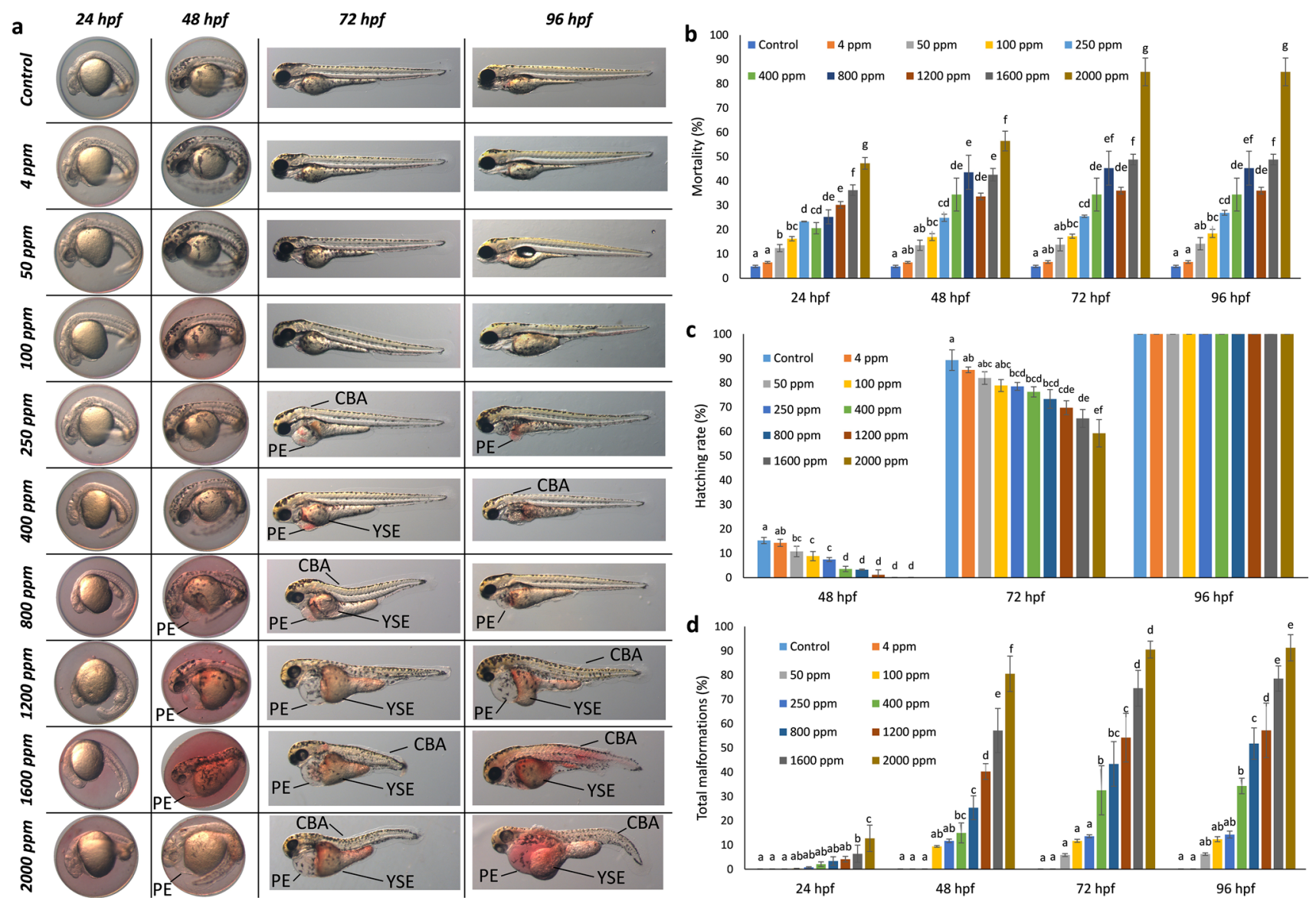

Fig. 1 Microscopic images of embryos exposed to carmoisine and control groups (a). The survival rate (b) and hatching rate (c) of zebrafish embryos during the exposure time of $96 \mathrm{~h}$. Distribution of observed body malformations (d). Data are expressed as

In this study, typical malformations including pericardial edema (PE), yolk sac edema (YSE), body malformations (BM) (including tail malformation, short tail and head malformation), and curved body axis (CBA) were examined as morphological endpoints. An increase in total malformation was observed in a dose-dependent manner (Fig. 1A, D). Mainly pericardial edema, yolk sac edema, tail anomaly and spinal curvature were observed. However, head malformation was not observed in larvae exposed to carmoisine. It has been reported that deformities or formation of large yolk sacs in zebrafish embryos may be related to delayed nutrient sac (yolk) resorption (Zoupa and Machera 2017). In addition, yolk sac edema is a common pathology observed in zebrafish developmental toxicity screenings. It is known that yolk is highly lipophilic and has low water content. The embryo maintains the osmotic balance in the water environment it is in, thanks to a water barrier in the yolk sac (Sant and Timme-Laragy 2018). It has been reported that azo dyes, due to their high water solubility properties, may disrupt this osmotic balance and cause excessive water intake into means \pm S.D. from three independent experiments $\left({ }^{*} p<0.05\right.$; ANOVA, Tukey's test) ( $P E$ pericardial edema, $Y S E$ yolk sac edema, CBA curved body axis)

the embryo and, as a result, edema formation (Jiang et al. 2020). Indeed, a study (Gupta et al. 2019) reported that a large yolk sac was observed in zebrafish embryos exposed to erythrosine which is another azo dye due to deformities or delayed bone development. The abnormality, spinal curvature and scoliosis observed in the larvae of the embryos exposed to carmoisine can be explained on the basis that this additive may have affected acetylcholine, acetylcholinesterase and receptor systems, thus neuronal and muscle development in zebrafish larvae (Behra et al. 2002). Spinal curvature and notochord bending have been reported in zebrafish larvae exposed to organophosphate chlorpyrifos, an acetylcholinesterase inhibitor (Sreedevi et al. 2014). Joshi and Katti (2018) observed tail distortion, yolk sac edema, and cardiac edema in zebrafish larvae exposed to another azo dye, tartrazine.

When evaluated in terms of apoptosis, it was found that the fluorescence intensity increased significantly in a dose-dependent manner, especially in larvae exposed to over 400 ppm of carmoisine (Fig. 2). Similar to our results, 


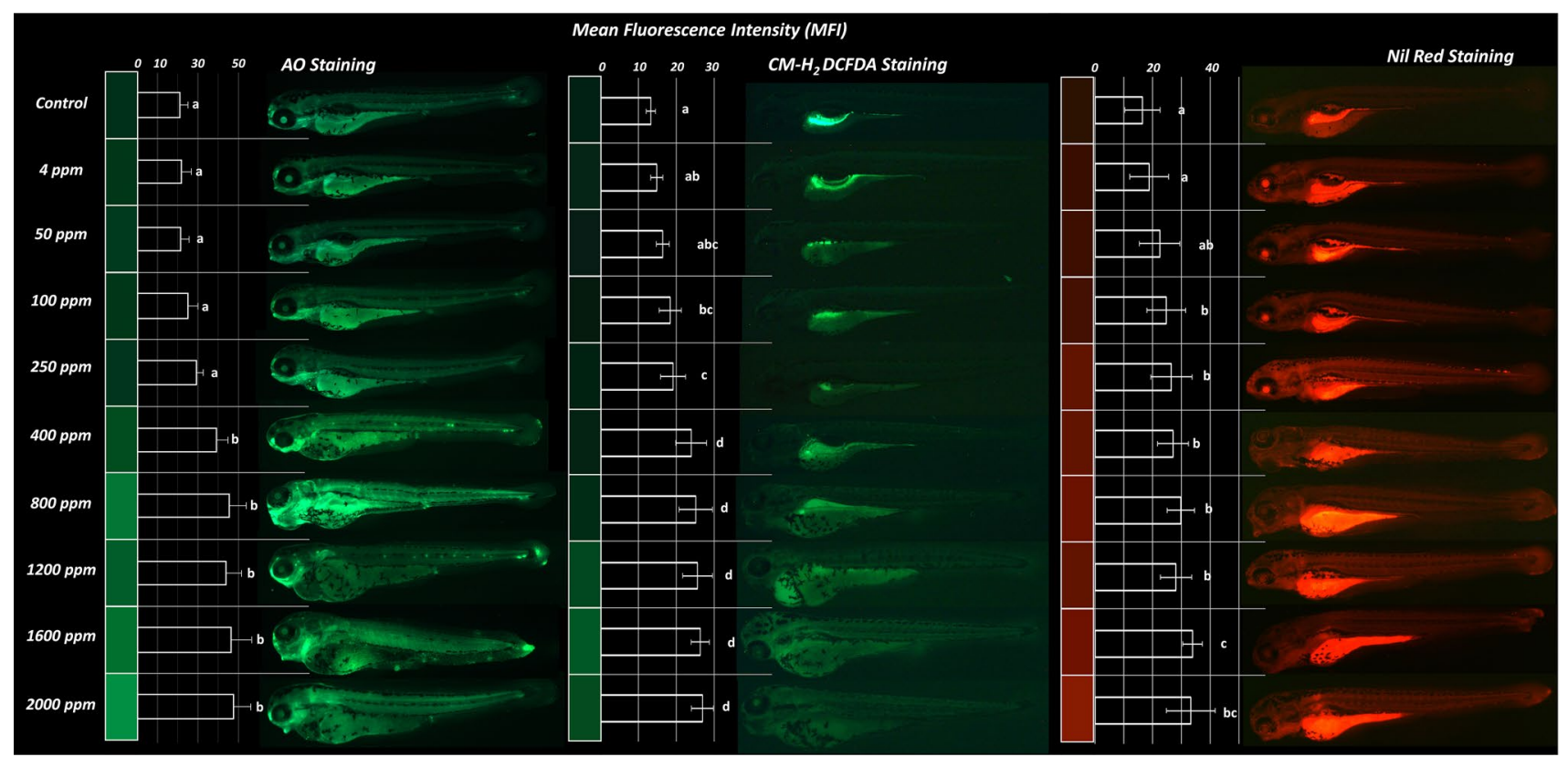

Fig. 2 Apoptotic cells were determined using acridine orange (AO) staining in $96 \mathrm{hpf}$ larvae and mean fluorescence intensity of the signals (Left). In vivo CM- $\mathrm{H}_{2}$ DCFDA staining of produced $\mathrm{ROS}$ in 96 hpf larvae and mean fluorescence intensity of the signals (Middle). Nile Red staining of adipocyte lipid droplets and mean fluorescence intensity of the signals (Right). To obtain more accurate results in

reactive black 5 azo dye has been reported to induce developmental defects in zebrafish embryos by enhancing apoptotic cell death (Manimaran et al. 2018). Moreover, different studies have suggested that exposure to synthetic textile dyes potentiates DNA damage and inhibits DNA repair through reactive oxygen species (ROS) generation and inhibition of DNA ligase (Liu et al. 2008). ROS is a term for a series of molecular oxygen derivatives that occur as a normal feature of aerobic life. ROS, which are kept in balance by different antioxidant defence systems in metabolism, cause molecular damage, referred to as 'oxidative stress', with high exposure to different triggering agents (Sies and Jones 2020). In this study, the level of ROS in live larvae exposed to carmoisine was evaluated by measuring mean fluorescence. The results revealed that the level of fluorescence intensity increased in a dose-dependent manner $(p<0.05)$ significantly in larvae exposed to carmoisine (Fig. 2). Indeed, it has been demonstrated that Acid Black, an azo dye, induced oxidative stress in freshwater Labeo rohita by Kaur and Kaur (Kaur and Kaur 2015).

Similarly, another azo dye, Disperse Red 1, activates antioxidant defenses to prevent LPO in freshwater Hydra attenuate (de Jong et al. 2016). In addition, it was determined that ROS overproduction and GST and superoxide dismutase activities were decreased in adult zebrafish exposed to textile industrial wastes (Zhang et al. 2012). fluorescence intensity analyses, larvae belonging to different groups were tried to be imaged in the same picture when imaging. Data are expressed as means \pm S.D. from three independent experiments (each replicate includes 5 larvae, ANOVA, $p<0.05$, Tukey's test) (color figure online)

Oxidative stress induced by azo dyes has also been noted to modulate genomic events, including oxidative stress gene-related transcriptional regulation, at early stages in the western frog (Silurana tropicalis) (Soriano et al. 2014).

It has been reported that xenobiotic compounds like food dyes taken with diets can induce obesity by altering endocrine signals and lipid hemostasis (Tingaud-Sequeira et al. 2011). Lipid metabolism can be altered in response to various factors inducing lipid accumulation, as a strategy to enable their growth and reproduction through cell division (D'Alessandro and Antoniosi Filho 2016). Nile Red is often preferred as a fluorescent probe to detect adipocyte lipid droplets in pharmaceutical or toxicological studies. In this study, zebrafish larvae exposed to carmoisine showed a dose-dependent increase in relative fluorescence intensity of Nile Red (Fig. 2). It is known that organisms exposed to most environmental stresses, including xenobiotic exposure, initiate regulatory processes through energy metabolism to maintain physiological homeostasis. It has been reported that serum total lipids, cholesterol, and triglycerides levels increased significantly in rats exposed to different doses of carmoisine orally (Aboel-Zahab et al. 1997). Elekima (2016) reported that total cholesterol, triglyceride, and LDL-Cas levels increased in rats exposed orally to carmoisine. The authors claimed that these increases may be due to the 
inability to produce enzymes such as lecithin cholesterol transferases and hepatic lipase, which are required for the degradation of cholesterol and triglycerides, respectively, due to hepatocyte damage caused by azo dyes.

In zebrafish, the heart is one of the first organs observed during the development stage. As indicators of heart function and physiological hemostasis, heart rate and blood flow rate are valuable parameters used to determine developmental toxicity in zebrafish. Results from this study revealed that exposure to carmoisine did not significantly affect heart rate during embryonic development of zebrafish, but caused a significant reduction in blood flow rate at the highest dose (Fig. 3). It has been reported that zebrafish can actively regulate heart rate in response to changes in the environment through branches of both the sympathetic and parasympathetic autonomic nervous systems (Li et al. 2017). In this regard, sustained exposure to azo dyes up to $120 \mathrm{hpf}$ has been reported to result in decreased embryonic heart rate (Stainier 2001). On the other hand, the significant reduction in blood flow rate observed at the highest dose may be associated with lower blood oxygen levels, indicative of increased hypoxanthine levels (Fig. 4). This molecule is mostly related to hypoxia (Lee et al. 2018).

According to the results, exposure to carmoisine caused a dose-dependent decrease in body length and eye size in zebrafish larvae (Fig. 3). It has been suggested in some

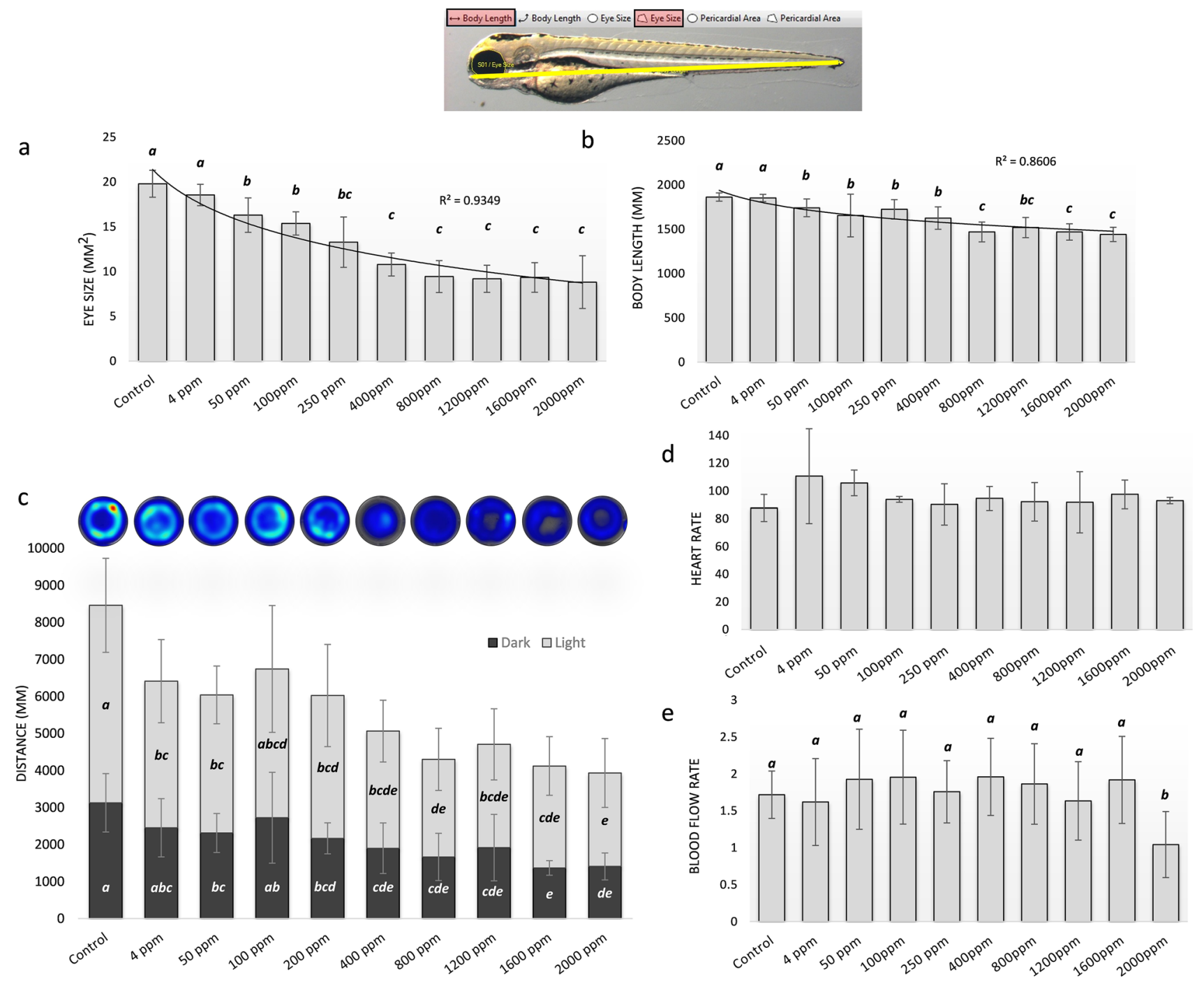

Fig. 3 Eye size (a) and body length (b) of carmoisine exposed and control zebrafish larvae at $5 \mathrm{dpf}$. Data are expressed as means \pm S.D. from three independent experiments (each replicate includes 20 larvae; $* p<0.05$; ANOVA, Tukey's test). Locomotor activity (total distance) (c) of carmoisine exposed and control zebrafish larvae at $6 \mathrm{dpf}$. Data are expressed as means \pm S.D. from three independent experi- ments (each replicate includes 24 larvae; $* p<.05$; ANOVA, Tukey's test). Heart rate (d) and blood flow (e) of carmoisine exposed and control zebrafish larvae at $5 \mathrm{dpf}$. Data are expressed as means \pm S.D. from three independent experiments (each replicate includes 10 larvae; ${ }^{*} p<0.05$; ANOVA, Tukey's test) 


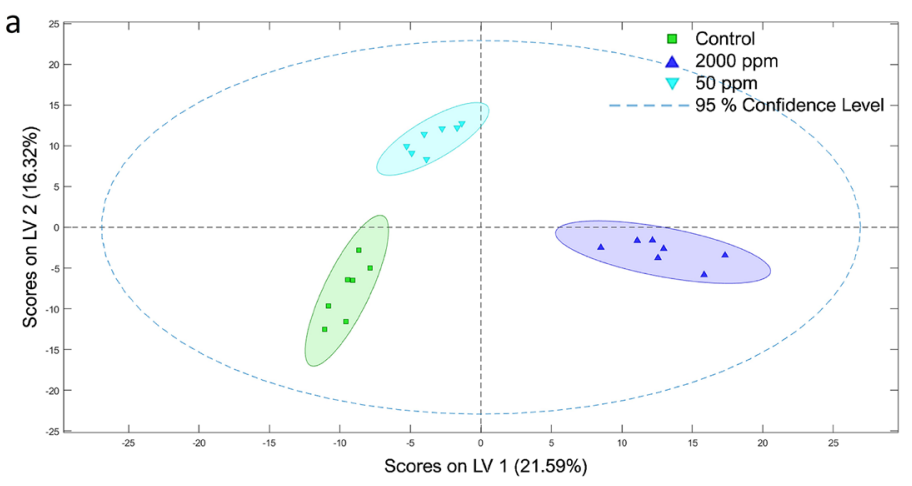

b

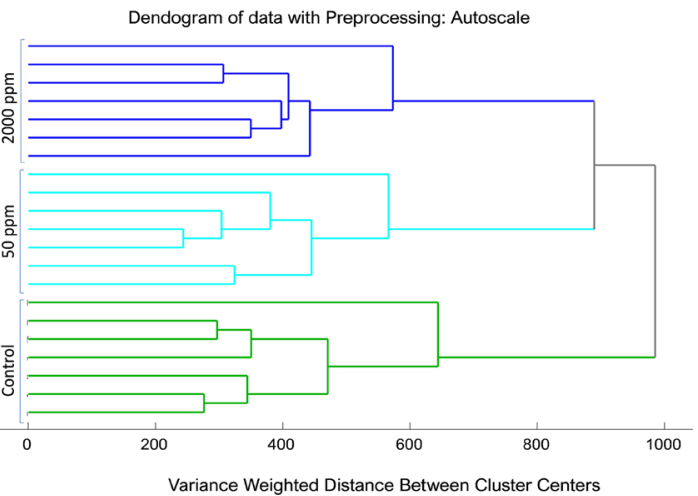

C

d

e
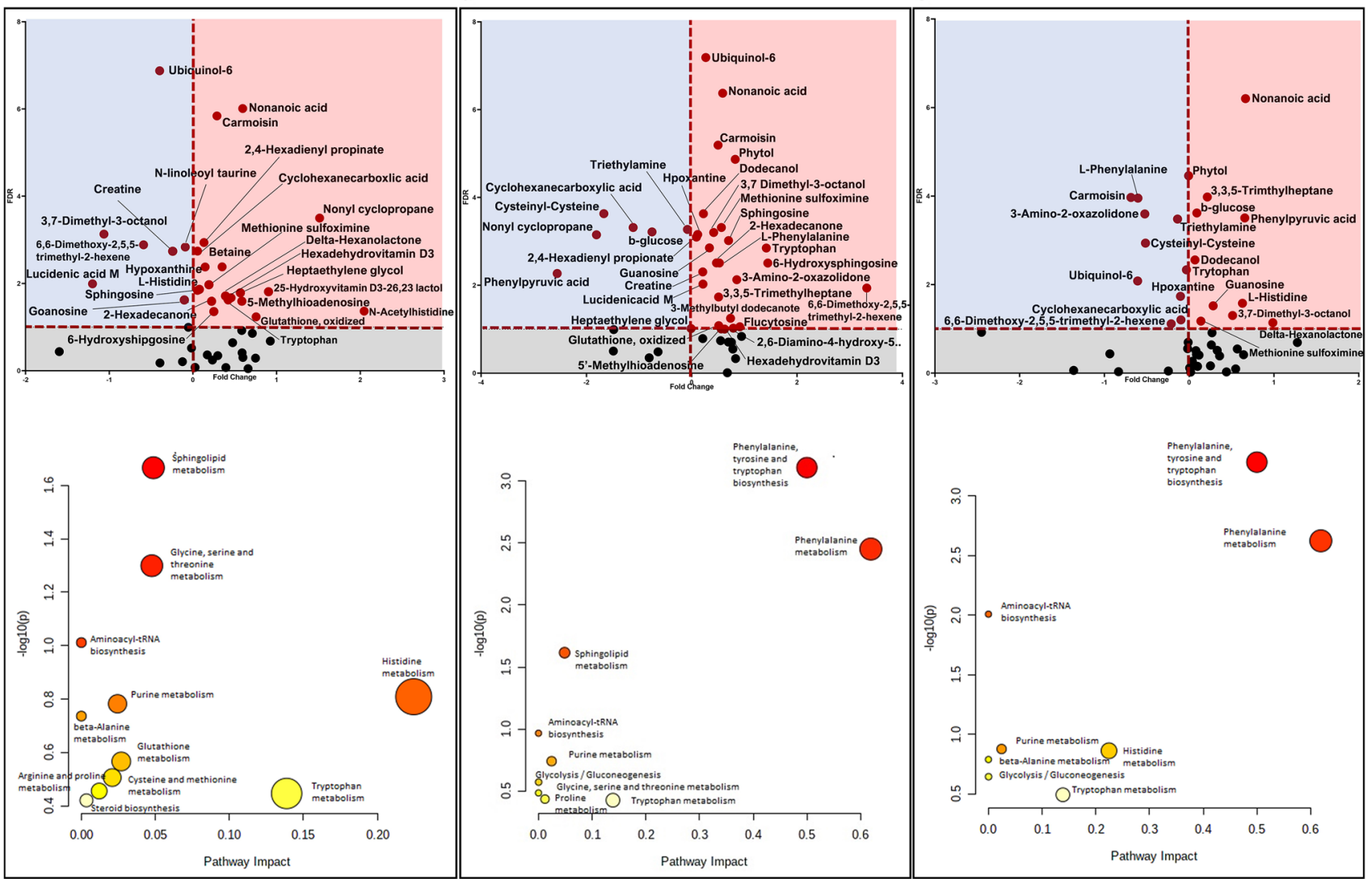

Fig. 4 The OPLS-DA scores plot for features (detected by TOF MS results) of control, $50 \mathrm{ppm}$ and $2000 \mathrm{ppm}$ exposure groups (a). Dendrogram of variance weighed distance between analyzed groups (b). Volcano graphs and pathway analysis plots of control vs 50 ppm (c), control vs $2000 \mathrm{ppm}$ (d) and $50 \mathrm{ppm}$ vs $2000 \mathrm{ppm}$ (e) carmoisine exposure including $\log 2$ (fold change) of the peak area of annotated metabolites and their $\log 10$ ( $p$ value). Upregulated (red) and down- regulated (blue) metabolites were monitored regarding to the carmoisine exposure in the volcano graph. The pathway analysis plots were created by Metaboanalyst 5.0. The results of pathway topological analysis were represented in $x$-axis as 'pathway impact' and logarithm of the $p$ value for pathway enrichment analysis were expressed in $y$-axis in accordance with annotated metabolites (color figure online) studies that it causes a decrease in the number of retinal cells with increased apoptosis, which causes the small eye phenotype. Consistent with our study, microphthalmia (reduction in eye diameter) was reported to be detected in zebrafish larvae exposed to Sunset Yellow (Joshi and Pancharatna 2019). It should not be overlooked that another reason for the decrease in body length may be increased malformations depending on the dose. Another significant impairment caused by carmoisine treatment was observed in locomotor activity (Fig. 3). Locomotor activity controlled at different levels of the nervous system is susceptible to many different endogenous/exogenous disturbances. Moreover, changes in locomotor activity can be regarded as indicative of changes in the neuromuscular junction, different central 
pattern makers, or more rostral levels (Dutra Costa et al. 2020). In this context, zebrafish larvae are recognized as an important model organism that can be used to evaluate different neurobehavioral domains in early life stages with ecologically relevant endpoints (Tegelenbosch et al. 2012). In current study, the behavioral effects of carmoisine were evaluated using zebrafish larvae. When Fig. $3 \mathrm{C}$ is examined, it has been seen that the control group larvae are clearly mobile both in total and in the night/day phases $(p<0.05)$. It can be said that this decrease in locomotor activity is in a trend dependent on carmoisine dose increase. No significant behavioral differences were detected in the larvae, including the control group, between the night and day phases. While this difference was more evident in the daytime phase, relatively similar findings were observed in the night phase.

The decreased locomotor activity observed in zebrafish larvae especially in high doses (between 800 and 2000 ppm) can be explained by two phenomena; first is dose-dependent increased malformations and second is the nervous system metabolome profile. It was stated above that an increasing malformation was observed depending on the dose of carmoisine exposure. In fact, studies have revealed that the malformations observed due to developmental toxicity in zebrafish larvae exposed to xenobiotics affect locomotor activity (Abe et al. 2017a, 2017b; Sulukan et al. 2021; To et al. 2021). The important thing at this point is to clarify the mechanism of action of the problems observed depending on the dose exposure. In this context, the current study has been investigated the metabolome profiles of zebrafish larvae exposed to high (2000 ppm) and low (50 ppm) doses of carmoisine. According to the metabolome results, remarkable alterations were detected in both low (50 ppm) concentration and high (2000 ppm) concentration of carmoisine exposure (Fig. 4). The analysis revealed 45 annotated metabolites and, as expected, the change in these metabolite levels was more dramatic at the higher concentration.

Guanosine is one of the annotated metabolites that found significantly high regulated in exposure groups. According to results, dose-dependent elevation has been observed in semi-quantitative intensity of Guanosine and approximately fivefold increase for $50 \mathrm{ppm}$ exposure group were measured against control group. This fold elevation reached about 10 in $2000 \mathrm{mg}$ exposure group. Guanosine is a purine nucleoside in cell metabolism and play an important role in neural degenerative injuries (Lanznaster et al. 2016). Due to its feature, dye exposure may induce its concentration to protect organism against the carmoisine toxicity. Second important metabolites are methionine sulfoximine $(\mathrm{MeS})$ which is known as the irreversible inhibitor of glutamine synthetase (Ronzio et al. 1969). Deficiency of glutamine synthetase may initiate pathological disorders as glial dysfunction, loss of neurons and abnormal movement in the living organisms (Zhou et al. 2019). It has been also reported that a high level of MeS may cause a low level of glutamine which is really important in brain function, especially in the neurotransmission of neuron cells (Albrecht et al. 2010). According to the results obtained from the histopathology analyzes, the degeneration and necrosis of the neurons, which increased in a dose-dependent manner, overlap with these findings (Fig. 5). Hypoxanthine which is mostly related to hypoxia is another annotated metabolite that was found significantly high in the carmoisine exposure groups with respect to control. Increasing amount of hypoxanthine indicate low level of oxygen in blood (Lee et al. 2018). This may change the cognitive behavior of any living organism. This increase can be explained as a result of oxygen deficiency due to the decrease in blood flow rate observed especially at the highest dose. Tryptophan is another molecule associated with hypoxia and neurodegenerative disorders. An increasing level of tryptophan may be an important sign for the neurodegenerative case (Platten et al. 2019). Phenylalanine and phenylpyruvic acid accumulation in the living organisms also cause neurological disorders (Asare et al. 2021). Therefore, the increase in the levels of these metabolites as a result of exposure to carmoisine may be a precursor to the differences in their behavior. N-Acetylhistidine and oxidized glutathione levels was also a sign of oxidative stress (Dwivedi et al. 2020; Holeček 2020) that may bring about neurodegenerative consequences on living organisms (Solleiro-Villavicencio and Rivas-Arancibia 2018). In addition to this, $\mathrm{N}$-acetyl-histidine, especially in fish, has a role in regulating the osmotic pressure of the eye as a water pump. This metabolic change may be the mechanism underlying the reduction in eye size observed as a result of exposure to carmoisine. According to this result, the dose-dependent reduction in eye size can be considered as a result of dehydration. On the other hand, sphingosine and 6-hydroxy sphingosine levels were found to be lower in carmoisine exposure groups. This situation was found in low-oxygen cases very similar with COVID19 and malaria (Marfia et al. 2021; Punsawad and Viriyavejakul 2017). These findings were compatible with the elevated levels of hypoxanthine and tryptophane.

In conclusion, high-dose exposure of carmoisine may cause low-oxygen levels, increasing oxidative stress and elevated level of some metabolites that cause neurodegenerative effects and behavioral alteration. Organism responds to this situation by increasing the concentration of antioxidant molecules as glutathione, $\mathrm{N}$-acetyl-histidine and neuroprotective agents like guanosine. It should also be taken into account that exposure to high doses of carmoisine may cause serious problems, and therefore, chronic exposures may cause the situation to be more dramatic. 

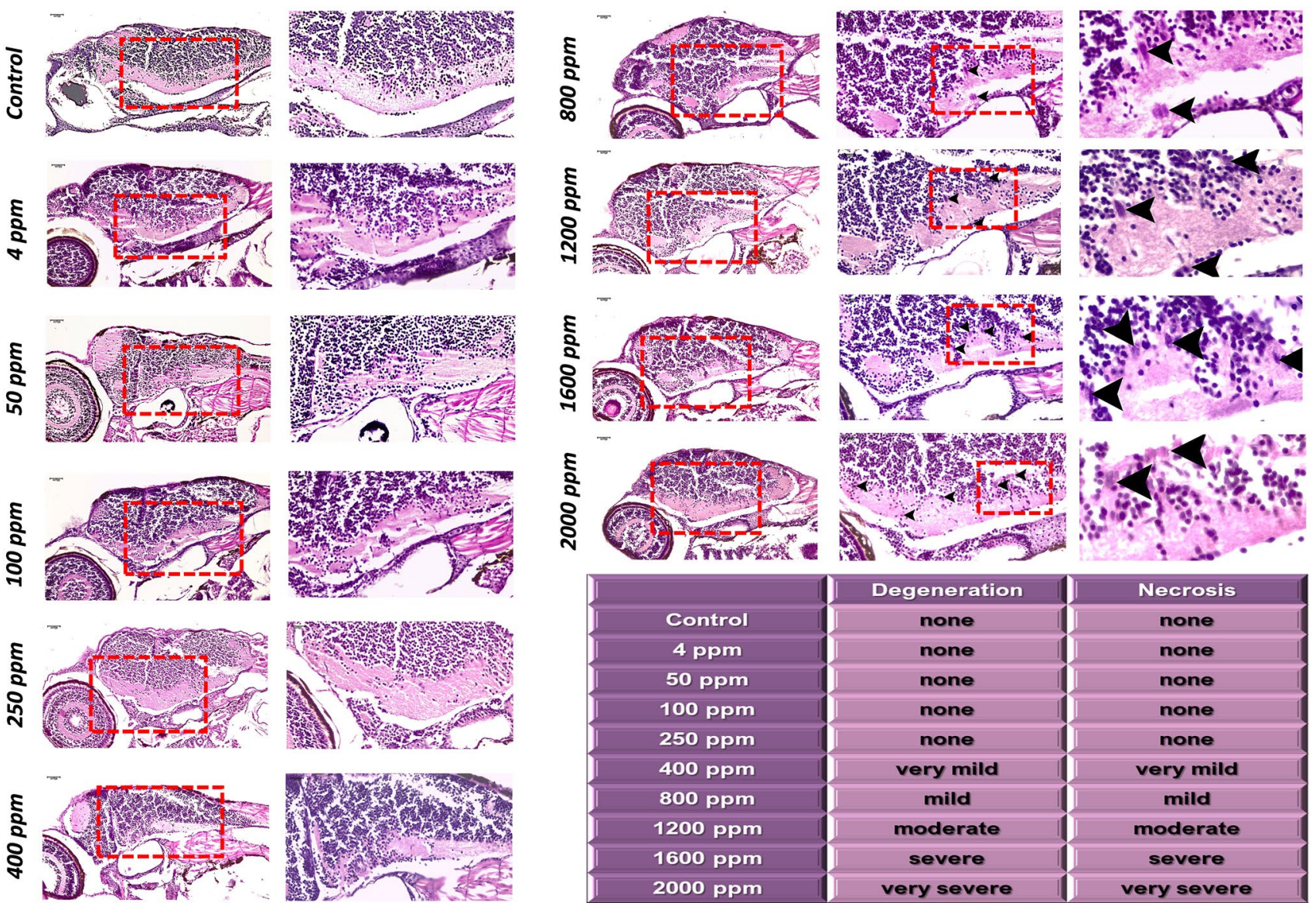

\begin{tabular}{|c|}
\hline Necrosis \\
\hline none \\
\hline none \\
\hline none \\
\hline none \\
\hline none \\
\hline very mild \\
\hline mild \\
\hline moderate \\
\hline severe \\
\hline very severe \\
\hline
\end{tabular}

Fig. 5 Microscopic image of hematoxylin and eosin staining ( $\mathrm{H}$ and $\mathrm{E}$ ) of zebrafish larvae exposed to carmoisine. Arrowhead indicates necrosis

Supplementary Information The online version contains supplementary material available at https://doi.org/10.1007/s00204-022-03240-2.

Acknowledgements We would like to thank YÖK (Council of Higher Education, Turkey) for the scholarship support given to Ekrem SULUKAN within the scope of the $100 / 2000 \mathrm{PhD}$ program. We also would like to thank DAYTAM (Eastern Anatolian High Technology Application Center) for instrument (Q-TOF MS/MS) support.

\section{Declarations}

Conflict of interest The authors declare that they have no conflict of interest.

\section{References}

Abe FR, Mendonça JN, Moraes LA et al (2017a) Toxicological and behavioral responses as a tool to assess the effects of natural and synthetic dyes on zebrafish early life. Chemosphere 178:282-290

Aboel-Zahab H, El-Khyat Z, Sidhom G, Awadallah R, Abdel-al W, Mahdy K (1997) Physiological effects of some synthetic food colouring additives on rats. Boll Chim Farm 136(10):615-627
Ai-Mashhedy LA, Fijer AN (2016) Acute toxicity of food additives tartrazine and carmoisine on white male mice. Int J PharmTech Res 9(4):364-367

Albrecht J, Sidoryk-Węgrzynowicz M, Zielińska M, Aschner M (2010) Roles of glutamine in neurotransmission. Neuron Glia Biol 6(4):263-276. https://doi.org/10.1017/S1740925X110000 93

Amin K, Hameid HA, AbdElsttar A (2010) Effect of food azo dyes tartrazine and carmoisine on biochemical parameters related to renal, hepatic function and oxidative stress biomarkers in young male rats. Food Chem Toxicol 48(10):2994-2999

Asare KK, Afrifa J, Opoku YK (2021) Mechanisms underlying induced pseudo-scleroderma among patients with phenylketonuria metabolic disorder. Global J Dermatol Venereol 9:1-8

Baran A, Köktürk M, Atamanalp M, Ceyhun SB (2018) Determination of developmental toxicity of zebrafish exposed to propyl gallate dosed lower than ADI (acceptable daily intake). Regul Toxicol Pharmacol 94:16-21. https://doi.org/10.1016/j.yrtph. 2017.12.027

Baran A, Yildirim S, Ghosigharehaghaji A, Bolat İ, Sulukan E, Ceyhun S (2020) An approach to evaluating the potential teratogenic and neurotoxic mechanism of BHA based on apoptosis induced by oxidative stress in zebrafish embryo (Danio rerio). Hum Exp Toxicol. https://doi.org/10.1177/0960327120952140

Behra M, Cousin X, Bertrand C et al (2002) Acetylcholinesterase is required for neuronal and muscular development in the zebrafish embryo. Nat Neurosci 5(2):111-118 
Capriello T, Visone IM, Motta CM, Ferrandino I (2021) Adverse effects of E150d on zebrafish development. Food Chem Toxicol 147:111877. https://doi.org/10.1016/j.fct.2020.111877

Chung KT, Stevens SE Jr, Cerniglia CE (1992) The reduction of azo dyes by the intestinal microflora. Crit Rev Microbiol 18(3):175190. https://doi.org/10.3109/10408419209114557

D’Alessandro EB, Antoniosi Filho NR (2016) Concepts and studies on lipid and pigments of microalgae: a review. Renew Sustain Energy Rev 58:832-841

de Jong L, Pech N, de Aragão UG, Moreau X (2016) Multi-scale biomarker evaluation of the toxicity of a commercial azo dye (disperse red 1) in an animal model, the freshwater cnidarian Hydra attenuata. Water Res 96:62-73

De la Paz JF, Beiza N, Paredes-Zúñiga S, Hoare MS, Allende ML (2017) Triazole fungicides inhibit zebrafish hatching by blocking the secretory function of hatching gland cells. Int J Mol Sci 18(4):710

Dutra Costa BP, Aquino Moura L, Gomes Pinto SA, Lima-Maximino M, Maximino C (2020) Zebrafish models in neural and behavioral toxicology across the life stages. Fishes. https://doi.org/10.3390/ fishes 5030023

Dwivedi D, Megha K, Mishra R, Mandal PK (2020) Glutathione in brain: overview of its conformations, functions, biochemical characteristics, quantitation and potential therapeutic role in brain disorders. Neurochem Res 45(7):1461-1480

Elbanna K, Sarhan OM, Khider M, Elmogy M, Abulreesh HH, Shaaban MR (2017) Microbiological, histological, and biochemical evidence for the adverse effects of food azo dyes on rats. J Food Drug Anal 25(3):667-680. https://doi.org/10.1016/j.jfda.2017.01.005

Elekima I (2016) Effect of carmoisine orally administered on lipid parameters of albino rats. Int J Sci Res 5(9):861-864

Gupta R, Ranjan S, Yadav A, Verma B, Malhotra K (2019) Toxic effects of food colorants erythrosine and tartrazine on zebrafish embryo development. Curr Res Nutr Food Sci J 7(3):876-885

Holeček M (2020) Histidine in health and disease: metabolism, physiological importance, and use as a supplement. Nutrients 12(3):848

Jiang L-L, Li K, Yan D-L, Yang M-F, Ma L, Xie L-Z (2020) Toxicity assessment of 4 azo dyes in zebrafish embryos. Int $\mathrm{J}$ Toxicol 39(2):115-123

Joshi V, Katti P (2018) Developmental toxicity assay for food additive tartrazine using zebrafish (Danio rerio) embryo cultures. Int $\mathbf{J}$ Toxicol 37(1):38-44

Joshi V, Pancharatna K (2019) Food colorant sunset yellow (E110) intervenes developmental profile of zebrafish (Danio rerio). J Appl Toxicol 39(4):571-581

Kaur S, Kaur A (2015) Variability in antioxidant/detoxification enzymes of Labeo rohita exposed to an azo dye, acid black (AB). Comp Biochem Physiol c: Toxicol Pharmacol 167:108-116

Khan IS, Ali MN, Hamid R, Ganie SA (2020) Genotoxic effect of two commonly used food dyes metanil yellow and carmoisine using Allium cepa L. as indicator. Toxicol Rep 7:370-375. https://doi. org/10.1016/j.toxrep.2020.02.009

Kiayi Z, Lotfabad TB, Heidarinasab A, Shahcheraghi F (2019) Microbial degradation of azo dye carmoisine in aqueous medium using Saccharomyces cerevisiae ATCC 9763. J Hazard Mater 373:608619. https://doi.org/10.1016/j.jhazmat.2019.03.111

Lanznaster D, Dal-Cim T, Piermartiri TCB, Tasca CI (2016) Guanosine: a neuromodulator with therapeutic potential in brain disorders. Aging Dis 7(5):657-679. https://doi.org/10.14336/AD. 2016.0208

Lee JS, Wang RX, Alexeev EE et al (2018) Hypoxanthine is a checkpoint stress metabolite in colonic epithelial energy modulation and barrier function. J Biol Chem 293(16):6039-6051

Li K, Wu J-Q, Jiang L-L et al (2017) Developmental toxicity of 2, 4-dichlorophenoxyacetic acid in zebrafish embryos. Chemosphere 171:40-48
Liu F, Gentles A, Theodorakis CW (2008) Arsenate and perchlorate toxicity, growth effects, and thyroid histopathology in hypothyroid zebrafish Danio rerio. Chemosphere 71(7):1369-1376

Manimaran D, Sulthana A, Elangovan N (2018) Reactive black 5 induced developmental defects via potentiating apoptotic cell death in zebrafish (Danio rerio) embryos. Pharm Pharmacol Int J 6(6):449-452

Marfia G, Navone S, Guarnaccia L et al (2021) Decreased serum level of sphingosine-1-phosphate: a novel predictor of clinical severity in COVID-19. EMBO Mol Med 13(1):e13424

Micheletti L, Coldibeli B, Salamanca-Neto CAR, Almeida LC, Sartori ER (2020) Assessment of the use of boron-doped diamond electrode for highly sensitive voltammetric determination of the azodye carmoisine E-122 in food and environmental matrices. Talanta 220:121417. https://doi.org/10.1016/j.talanta.2020.121417

OECD (2013), Test No. 236: Fish Embryo Acute Toxicity (FET) Test, OECD Guidelines for the Testing of Chemicals, Section 2, OECD Publishing, Paris. https://doi.org/10.1787/9789264203709-en

Peksa V, Jahn M, Štolcová L et al (2015) Quantitative SERS analysis of azorubine (E 122) in sweet drinks. Anal Chem 87(5):2840-2844. https://doi.org/10.1021/ac504254k

Platten M, Nollen EAA, Röhrig UF, Fallarino F, Opitz CA (2019) Tryptophan metabolism as a common therapeutic target in cancer, neurodegeneration and beyond. Nat Rev Drug Discov 18(5):379401. https://doi.org/10.1038/s41573-019-0016-5

Punsawad C, Viriyavejakul P (2017) Reduction in serum sphingosine 1-phosphate concentration in malaria. PLoS ONE 12(6):e0180631

Reza MSA, Hasan MM, Kamruzzaman M et al (2019) Study of a common azo food dye in mice model: toxicity reports and its relation to carcinogenicity. Food Sci Nutr 7(2):667-677. https://doi.org/ 10.1002/fsn3.906

Ronzio RA, Rowe WB, Meister A (1969) Mechanism of inhibition of glutamine synthetase by methionine sulfoximine. Biochemistry 8(3):1066-1075

Sant KE, Timme-Laragy AR (2018) Zebrafish as a model for toxicological perturbation of yolk and nutrition in the early embryo. Curr Environ Health Rep 5(1):125-133

Sies H, Jones DP (2020) Reactive oxygen species (ROS) as pleiotropic physiological signalling agents. Nat Rev Mol Cell Biol 21(7):363383. https://doi.org/10.1038/s41580-020-0230-3

Sohrabi MR, Khavaran A, Shariati S, Shariati S (2017) Removal of Carmoisine edible dye by Fenton and photo Fenton processes using Taguchi orthogonal array design. Arab J Chem 10:S3523 S3531. https://doi.org/10.1016/j.arabjc.2014.02.019

Sökmen TÖ, Sulukan E, Türkoğlu M, Baran A, Özkaraca M, Ceyhun SB (2020) Polystyrene nanoplastics $(20 \mathrm{~nm})$ are able to bioaccumulate and cause oxidative DNA damages in the brain tissue of zebrafish embryo (Danio rerio). Neurotoxicology 77:51-59

Solleiro-Villavicencio H, Rivas-Arancibia S (2018) Effect of chronic oxidative stress on neuroinflammatory response mediated by CD4+ T cells in neurodegenerative diseases. Front Cell Neurosci $12: 114$

Soriano JJ, Mathieu-Denoncourt J, Norman G, de Solla SR, Langlois VS (2014) Toxicity of the azo dyes acid red 97 and Bismarck brown Y to western clawed frog (Silurana tropicalis). Environ Sci Pollut Res 21(5):3582-3591

Sreedevi B, Suvarchala G, Philip GH (2014) Morphological and physiological abnormalities during development in zebrafish due to chlorpyrifos. Indian J Sci Res 5(2):1-8

Stainier DY (2001) Zebrafish genetics and vertebrate heart formation. Nat Rev Genet 2(1):39-48

Sulukan E, Ghosigharehagaji A, Baran A, Yildirim S, Bolat İ, Ceyhun SB (2021) A versatile toxicity evaluation of ethyl carbamate (urethane) on zebrafish embryos: morphological, physiological, histopathological, immunohistochemical, transcriptional and 
behavioral approaches. Toxicol Lett 353:71-78. https://doi.org/ 10.1016/j.toxlet.2021.09.012

Suvarchala G, Philip GH (2016) Toxicity of 3, 5, 6-trichloro-2-pyridinol tested at multiple stages of zebrafish (Danio rerio) development. Environ Sci Pollut Res 23(15):15515-15523

Tegelenbosch RAJ, Noldus LPJJ, Richardson MK, Ahmad F (2012) Zebrafish embryos and larvae in behavioural assays. Behaviour 149(10-12):1241-1281. https://doi.org/10.1163/1568539X-00003 020

Tingaud-Sequeira A, Ouadah N, Babin PJ (2011) Zebrafish obesogenic test: a tool for screening molecules that target adiposity. J Lipid Res 52(9):1765-1772

ToSt. MaryWooley KTLAH et al (2021) Morphological and behavioral effects in zebrafish embryos after exposure to smoke dyes. Toxics 9(1):9

Türkoğlu M, Baran A, Sulukan E et al (2021) The potential effect mechanism of high-fat and high-carbohydrate diet-induced obesity on anxiety and offspring of zebrafish. Eat Weight Disord-Stud Anorex, Bulim Obes. https://doi.org/10.1007/s40519-021-01140-5
Zhang W, Liu W, Zhang J et al (2012) Characterisation of acute toxicity, genotoxicity and oxidative stress posed by textile effluent on zebrafish. J Environ Sci 24(11):2019-2027

Zhou Y, Dhaher R, Parent M et al (2019) Selective deletion of glutamine synthetase in the mouse cerebral cortex induces glial dysfunction and vascular impairment that precede epilepsy and neurodegeneration. Neurochem Int 123:22-33

Zoupa M, Machera K (2017) Zebrafish as an alternative vertebrate model for investigating developmental toxicity - the triadimefon example. Int J Mol Sci 18(4):817

Publisher's Note Springer Nature remains neutral with regard to jurisdictional claims in published maps and institutional affiliations. 